\title{
Effects of Gamma Irradiation of Human Serum Samples from rVSVAG-ZEBOV-GP (V920) Ebola Virus Vaccine Recipients on Plaque-Reduction Neutralization Assays
}

\author{
Rebecca J. Grant-Klein, ${ }^{1}$ Joseph Antonello, ${ }^{1}$ Rick Nichols, ${ }^{2}$ Sheri Dubey, ${ }^{1}$ and Jakub K. Simon ${ }^{1 *}$ \\ ${ }^{1}$ Merck \& Co., Inc., Kenilworth, New Jersey; ${ }^{2}$ Crozet BioPharma, Devens, Massachusetts
}

\begin{abstract}
Gamma irradiation (GI) is included in the CDC guidance on inactivation procedures to render a group of select agents and toxins nonviable. The Ebola virus falls within this group because it potentially poses a severe threat to public health and safety. To evaluate the impact of $\mathrm{Gl}$ at a target dose of $50 \mathrm{kGy}$ on neutralizing antibody titers induced by the rVSVAG-ZEBOV-GP vaccine (V920), we constructed a panel of 48 paired human serum samples (GI-treated versus non-GI-treated) from healthy participants selected from a phase 3 study of V920 (study V920-012; NCT02503202). Neutralizing antibody titers were determined using a validated plaque-reduction neutralization test. Gl of sera from V920 recipients was associated with approximately $20 \%$ reduction in postvaccination neutralizing antibody titers. Gl was not associated with any change in pre-vaccination neutralizing antibody titers.
\end{abstract}

\section{INTRODUCTION}

Recombinant vesicular stomatitis virus-Zaire Ebola virus envelope glycoprotein (rVSVAG-ZEBOV-GP) vaccine (V920) demonstrated robust immunogenicity in early-phase studies in Europe, North America, and Africa in countries unaffected by the 2013-2016 Ebola outbreak. ${ }^{1-4}$ Robust vaccine immunogenicity was also observed in phase $2 / 3$ studies in African countries directly affected by the outbreak, ${ }^{5,6}$ where one phase 3 study demonstrated vaccine efficacy. ${ }^{7}$

In 2019, the European Medicines Agency granted conditional marketing authorization for V920 (ERVEBO ${ }^{\circledR}$, Merck \& Co., Inc., Kenilworth, NJ; November 11), ${ }^{8}$ prompting WHO prequalification (November 12) ${ }^{9}$ and Food and Drug Administration approval (December 20). ${ }^{10}$ In Africa, V920 has been registered by health authorities within the Democratic Republic of the Congo, Burundi, Ghana, and Zambia; efforts to obtain licensure for V920 use in additional African countries affected by Ebola virus are ongoing. ${ }^{11}$

The CDC guidance on inactivation procedures to render select agents nonviable includes gamma irradiation (Gl) and outlines the need for validated processes suitable for clinical samples potentially infected with select agents before shipping, processing, and clinical testing. ${ }^{12}$ Given its potential as a severe threat to public health and safety, Ebola virus falls within this group of biological select agents and toxins.

Two independent, prospective studies evaluated the Gl effect (target dose: $50 \mathrm{kGy}$ ) on antibodies against recombinant ZEBOV GP in a validated ELISA, using pre- and post-V920 vaccination serum samples from participants in a North American phase 1 clinical study. ${ }^{13}$ GI resulted in approximately $20 \%$ higher and $20 \%$ lower antibody concentrations in pre- and postvaccination samples, respectively. We concluded the decreased probability of transmitting viable Ebola virus to testing personnel far outweighs the effect on antibody titer.

In this study, we evaluated the Gl impact (same target dose of 50 kGy) on neutralizing antibody titers in human serum samples from a phase 3 study conducted in North America and Europe, as assessed by a validated plaque-reduction neutralization test (PRNT).

*Address correspondence to Jakub K. Simon, Merck \& Co., Inc., 2000 Galloping Hill Road, Kenilworth, NJ 07033. E-mail: jakub.simon@ merck.com

\section{METHODS}

This prospective study investigated any measurable change in neutralizing antibody in serum following Gl treatment versus non-Gl-treated aliquots using validated PRNT. A panel of paired samples from 48 participants (Gl-treated versus non-GItreated; 96 total samples) from a phase 3 study of the V920 vaccine in healthy adults from the United States, Canada, and Spain (NCT02503202) ${ }^{14,15}$ was evaluated. Samples were selected to provide a broad range of neutralizing antibody titers, based on their historic PRNT result, and included 12 historic pre-vaccination and 36 historic postvaccination samples (prior PRNT performed by $\mathrm{Q}^{2}$ Solutions [San Juan Capistrano, CA]; Supplemental Table 1).

Full details on sample preparation, handling, and shipping were described previously. ${ }^{13}$ In brief, serum samples were prepared at Merck \& Co., Inc. testing lab (West Point, PA) before shipping and/or treatment. Duplicate tubes from each sample were placed in separate boxes (Supplemental Figure 1); Box 1 was shipped on dry ice to Sterigenics (Corona, CA) for GI treatment at a target dose of $50 \mathrm{kGy}$, as described previously, ${ }^{13}$ and subsequently shipped on dry ice to $Q^{2}$ Solutions. A replicate untreated box (Box 2) was shipped directly to $Q^{2}$ Solutions on dry ice.

Neutralizing antibody titers were measured at $Q^{2}$ Solutions using the same PRNT reported previously. ${ }^{16}$ The assay uses rVSV $\triangle$ G-ZEBOV-GP as the target virus, and was developed and validated to quantify neutralizing antibodies postV920 vaccination.

Serum was serially diluted and mixed with an equal volume of live rVSV $\triangle$ G-ZEBOV-GP for final dilutions (range: 1:10-1:20, 480). Internal quality controls were included. Neutralization proceeded over 18 hours at $2-8^{\circ} \mathrm{C}$, after which the serum/virus mixture was used to inoculate Vero cells. Viral adsorption was performed at $37 \pm 2^{\circ} \mathrm{C}$ for 60 minutes, after which a methylcellulose overlay was added. Infected cells were incubated at $37 \pm 2^{\circ} \mathrm{C} 5 \% \mathrm{CO}_{2}$ for 2 days. Plaques were visualized by crystal violet stain and counted automatically using an AID Reader (Autoimmun Diagnostika GmbH, Strassberg, Germany); neutralizing titers were calculated based on the percent reduction in viral plaques in the presence of serum versus virus control without serum. Results are reported as plaquereduction neutralization of $60 \%\left(\mathrm{PRNT}_{60}\right)$, with the reciprocal serum dilution resulting in a $60 \%$ reduction in plaque number. The PRNT has completed validation, and the report has 
undergone Center for Biologics Evaluation and Research review. $\mathrm{PRNT}_{60}$ was used because of improved total assay precision over a less conservative endpoint of plaque-reduction neutralization of $50 \%$ (PRNT 50 ).

Geometric mean fold differences in titer between $\mathrm{Gl}$ and non-Gl and $95 \%$ Cls were estimated and statistically assessed using a paired $t$-test. The correlation between assay measures was estimated by the Pearson correlation coefficient and Lin's coefficients for accuracy and concordance, respectively. ${ }^{17,18}$ To assess if $\mathrm{Gl}$ effect is dependent on the titer level, the natural log-transformed Gl titers were regressed against the natural log-transformed non-Gl titers using errors-in-variables regression. ${ }^{19}$

Qualitative comparisons between assay methods were based on $2 \times 2$ cross-classification tables about the PRNT limit of detection (LOD) of 20 . From the $2 \times 2$ cross-classification tables, the agreement rate (proportion of double-positive and double-negative samples relative to the total number of samples) was reported. The $95 \% \mathrm{Cl}$ for the estimated agreement rate was computed based on the binomial distribution, and imbalance in the distribution of discordant samples was assessed using an exact McNemar's test.

\section{RESULTS}

Figure 1 displays the V920 PRNT titers, and Table 1 shows the statistical comparisons between antibody titers of Gl-treated samples and corresponding non-GI samples. On average, Gl was associated with a small, but statistically significant, reduction (approximately 20\%) in postvaccination PRNT titers (1.19-fold reduction; 95\% Cl: 1.06-1.34). Fold reduction in titer and its $95 \% \mathrm{Cl}$ are the reciprocals of the geometric mean fold ratio in titer (Gl/non-Gl) and its $95 \% \mathrm{Cl}$ (Table 1). The proximity of the estimated concordance slope to
1.0 (95\% Cl: $0.85-1.09)$ suggests the effect is fairly constant throughout the positive titer range evaluated. The closeness of Pearson's correlation coefficient (0.94), Lin's accuracy coefficient (0.98), and Lin's concordance correlation coefficient (0.93) to 1.0 provides additional support that $\mathrm{Gl}$ effect is small and consistent.

Qualitative comparisons between the two procedures used for $\mathrm{Gl}$ and non-Gl sample sets showed little discordance. Based on the $2 \times 2$ cross-classification table about the PRNT LOD of 20 , all 36 of the historic postvaccination samples had detectable titers (i.e., were positive) in both GI and non-GI conditions (Supplemental Table 2). Similarly, none of the 12 historic pre-vaccination samples had detectable titers (i.e., no negative samples became positive with $\mathrm{Gl}$ ).

\section{DISCUSSION}

We have described a controlled inactivation procedure enabling the high-throughput treatment of potentially infectious clinical specimens before performing immunogenicity assays essential to support clinical trials in a vaccine program.

Our results suggest Gl of pre-vaccination samples is not associated with detectable changes in neutralizing antibody titers, as measured in the validated PRNT; none of the 12 nonGI samples with neutralizing antibody titers less than the PRNT LOD had detectable titers following GI. Furthermore, Gl of postvaccination sera across a wide range of titers was associated with consistently minor decreases in neutralizing antibody titers. Results are consistent with the previously reported effect on antibody concentrations using ELISA with postvaccination antibody-positive sera. ${ }^{13}$

An explanation for why antibodies in pre-vaccination samples are increased with Gl for ELISA but not PRNT while measurements in postvaccination samples are decreased

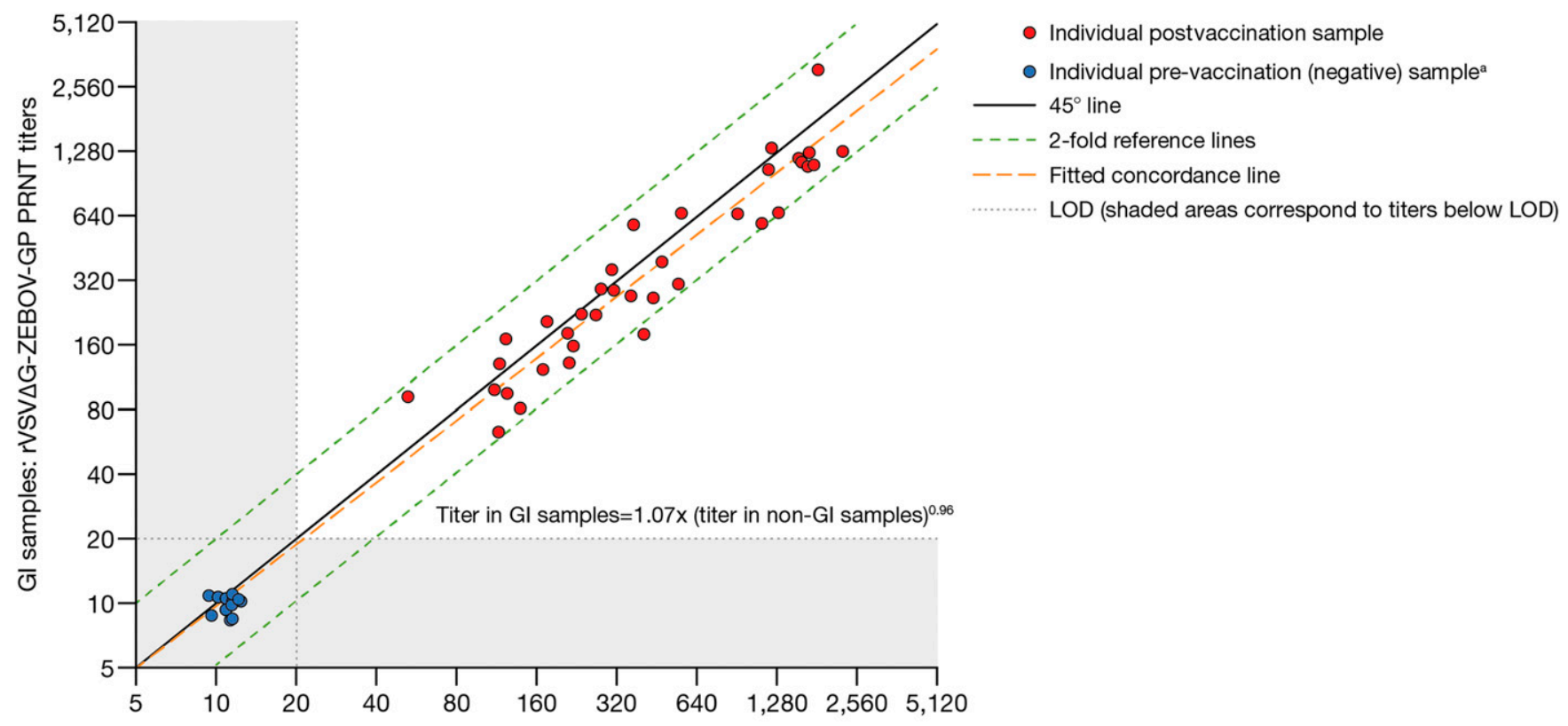

Non-GI samples: rVSVAG-ZEBOV-GP PRNT titers

FIGURE 1. Concordance plot displaying the effect of GI on neutralizing antibody titer of human clinical samples from vaccinated subjects as measured in the V920 PRNT. ${ }^{a}$ For ease of data visualization, pre-vaccination samples (titers $<20$ ), which were excluded from the analyses, were randomly assigned a titer close to $10 . \mathrm{GI}=$ gamma irradiation; $L O D=$ limit of detection; PRNT = plaque-reduction neutralization test; rVSV $\Delta$ G-ZEBOV-GP = recombinant vesicular stomatitis virus-Zaire Ebola virus envelope glycoprotein; V920 = rVSV $\Delta \mathrm{G}-Z \mathrm{EBOV}-\mathrm{GP}$ vaccine. 
TABLE 1

Summary measures of agreement between $\mathrm{Gl}$ and non-Gl procedures

\begin{tabular}{llcr}
\hline \multicolumn{1}{c}{ Concordance parameter } & $N$ & Estimate or $P$-value & $95 \%$ Cl \\
\hline Serostatus agreement (\%) & \multirow{2}{*}{48} & 100 & $92.6-100$ \\
Discordance imbalance $P$-value & & 0.0000 & $0.75-0.95$ \\
\hline Geometric mean fold ratio in titer(Gl/non-Gl) & & 0.94 & $0.89-0.97$ \\
Pearson's correlation coefficient & \multirow{2}{*}{36} & 0.98 & $0.97-0.99$ \\
Lin's accuracy coefficient & & 0.96 & $0.87-0.96$ \\
Lin's concordance correlation coefficient & & 1.07 & $0.85-1.09$ \\
Concordance slope & & & $0.51-2.26$ \\
Concordance intercept & &
\end{tabular}

with GI for both ELISA and PRNT is that for ELISA, GI may break down cellular debris or cause nonspecific antibodies, present in all normal human sera, to fragment. Without competition from specific rVSVIG-ZEBOV-GP antibodies, debris or fragments could nonspecifically bind ZEBOV-GP coating antigen, producing a false-positive signal above lower limit of quantitation. In postvaccination sera, nonspecific binding would be overwhelmed by specific antibodies with higher affinity, some of which are also broken down by Gl, as evidenced by slightly impaired specific binding. Plaque-reduction neutralization test, however, measures functional neutralizing antibody not expected to have nonspecific activity, and only demonstrates loss of specific activity after Gl.

As described previously, ${ }^{13}$ study limitations include use of specimens from North American and European subjects with minimal pre-vaccination antibody levels; potential GI impact on sera from subjects living in regions where Ebola circulates, who may have higher baseline antibody titers, was not evaluated. Future studies using sera from subjects with possible current or prior wild-type Ebola virus infections would require appropriate biosafety level (BSL) laboratory conditions.

This Gl study may support future vaccine trials in regions where BSL 3/4 organisms require sample testing. Gl may provide additional safety for laboratory personnel against lethal pathogens present in study samples. ${ }^{12}$ Current and prior results indicate $\mathrm{Gl}$ effect is small and consistent across a range of antibody titers. ${ }^{13}$ Assuming all study samples receive the same Gl treatment, our results suggest Gl effect is unlikely to bias treatment comparisons within a study.

\section{CONCLUSION}

Gl of V920-vaccinated subject sera was associated with approximately $20 \%$ reduced neutralizing antibody titers as measured by PRNT postvaccination and was not associated with any change in neutralizing antibody titers pre-vaccination.

$\mathrm{Gl}$ is recommended to reduce the transmission of viable Ebola virus to testing personnel when specimens may contain wild-type virus. Any Gl impact on immunogenicity should be considered when interpreting PRNT data.

Received August 20, 2020. Accepted for publication January 24, 2021.

Published online March 29, 2021.

Note: Supplemental tables and figure appear at www.ajtmh.org.
Acknowledgments: We would like to thank the clinical study participants and Kelli Clifton from the CDC for their advice regarding irradiation dose. In addition, we also thank the CDC Sierra Leone Trial to Introduce a Vaccine Against Ebola (STRIVE) team; the following colleagues from $\mathrm{Q}^{2}$ Solutions: Wayne Hogrefe, Gary Peterson, Brent Seaton, Janine Naguiat, and Sara Daijogo; and the Biomedical Advanced Research and Development Authority.

Financial support: This study was funded by Merck Sharp \& Dohme Corp., a subsidiary of Merck \& Co., Inc., Kenilworth, NJ. This project has been funded in part with Federal funds from the Department of Health and Human Services; Office of the Assistant Secretary for Preparedness and Response; and Biomedical Advanced Research and Development Authority, under contract no. HHSO100201500002C. Medical writing assistance, under the direction of the authors, was provided by Adele Blair, of CMC AFFINITY, McCann Health Medical Communications, in accordance with Good Publication Practice (GPP3) guidelines. This assistance was funded by Merck Sharp \& Dohme Corp., a subsidiary of Merck \& Co., Inc., Kenilworth, NJ.

Disclosure: R. J. G.-K., J. A., S. D., and J. K. S. are employees of Merck Sharp \& Dohme Corp., a subsidiary of Merck \& Co., Inc., Kenilworth, $\mathrm{NJ}$, and may own stock/stock options in Merck \& Co., Inc., Kenilworth, NJ. R. N. has received personal fees from New Link Genetics as a consultant.

Authors' addresses: Rebecca J. Grant-Klein, Joseph Antonello, Sheri Dubey, and Jakub K. Simon, Merck \& Co., Inc., Kenilworth, NJ, E-mails: rebecca.klein1@merck.com, joseph_antonello@merck. com, sheri_dubey@merck.com, and jakub.simon@merck.com. Rick Nichols, Crozet BioPharma, Devens, MA, E-mail: rick.nichols@ crozetbiopharma.com.

\section{REFERENCES}

1. Agnandji ST et al., 2016. Phase 1 trials of rVSV Ebola vaccine in Africa and Europe. N Engl J Med 374: 1647-1660.

2. ElSherif MS et al., 2017. Assessing the safety and immunogenicity of recombinant vesicular stomatitis virus Ebola vaccine in healthy adults: a randomized clinical trial. CMAJ 189: E819-E827.

3. Regules JA et al., 2017. A recombinant vesicular stomatitis virus Ebola vaccine. N Engl J Med 376: 330-341.

4. Huttner A et al., 2015. The effect of dose on the safety and immunogenicity of the VSV Ebola candidate vaccine: a randomised double-blind, placebo-controlled phase 1/2 trial. Lancet Infect Dis 15: 1156-1166.

5. Kennedy SB et al., 2017. Phase 2 placebo-controlled trial of two vaccines to prevent Ebola in Liberia. $N$ Engl $J$ Med 377: 1438-1447.

6. Samai $\mathrm{M}$ et al., 2018. The Sierra Leone trial to introduce a vaccine against Ebola: an evaluation of rVSV $\triangle$ G-ZEBOV-GP vaccine tolerability and safety during the west Africa Ebola outbreak. $J$ Infect Dis 217 (Suppl 1): S6-S15.

7. Henao-Restrepo AM et al., 2017. Efficacy and effectiveness of an rVSV-vectored vaccine in preventing Ebola virus disease: final results from the Guinea ring vaccination, open-label, clusterrandomised trial (Ebola Ca Suffit!). Lancet 389: 505-518. 
8. European Medicines Agency (EMA), 2019. Summary of Opinion (Initial Authorisation): Ervebo Ebola Zaire Vaccine (rVSVAGZEBOV-GP, Live). Available at: https://www.ema.europa.eu/ en/documents/smop-initial/chmp-summary-positive-opinionervebo_en.pdf. Accessed June 12, 2020.

9. World Health Organization, 2019. WHO prequalifies Ebola vaccine, paving the way for its use in high-risk countries. Geneva, Switzerland: WHO. Available at: https://www. who.int/news-room/detail/12-11-2019-who-prequalifiesebola-vaccine-paving-the-way-for-its-use-in-high-risk-countries. Accessed June 12, 2020

10. Merck \& Co., Inc., Kenilworth, NJ, 2019. Merck Announces FDA Approval for ERVEBO ${ }^{\circledR}$ (Ebola Zaire Vaccine, Live). Available at: https://www.mrknewsroom.com/news-release/ebola/merckannounces-fda-approval-ervebo-ebola-zaire-vaccine-live. Accessed June 12, 2020.

11. Merck \& Co., Inc., Kenilworth. NJ, 2020. ERVEBO ${ }^{\circledR}$ (Ebola Zaire Vaccine, Live) Now Registered in Four African Countries, Within 90 Days of Reference Country Approval and WHO Prequalification. Available at: https://www.mrknewsroom.com/ news-release/ebola/ervebo-ebola-zaire-vaccine-live-nowregistered-four-african-countries-within-90-d. Accessed June 12, 2020.

12. Centers for Disease Control and Prevention, Division of Select Agents and Toxins, Animal and Plant Health Inspection Service, Agricultural Select Agent Program, 2017. Guidance on the Inactivation or Removal of Select Agents and Toxins for Future
Use. Available at: https://www.selectagents.gov/resources/ Inactivation_Guidance.pdf. Accessed June 12, 2020.

13. Grant-Klein RJ, Antonello J, Nichols R, Dubey S, Simon J, 2019. Effect of gamma irradiation on the antibody response measured in human serum from subjects vaccinated with recombinant vesicular stomatitis virus-zaire Ebola virus envelope glycoprotein vaccine. Am J Trop Med Hyg 101: 207-213.

14. Halperin SA et al., 2017. Six-month safety data of recombinant vesicular stomatitis virus-Zaire Ebola virus envelope glycoprotein vaccine in a phase 3 double-blind, placebo-controlled randomized study in healthy adults. J Infect Dis 215: 1789-1798.

15. Halperin SA et al., 2019. Immunogenicity, lot consistency, and extended safety of rVSV $\triangle$ G-ZEBOV-GP vaccine: a phase 3 randomized, double-blind, placebo-controlled study in healthy adults. J Infect Dis 220: 1127-1135.

16. Heppner DG, Jr et al., 2017. Safety and immunogenicity of the rVSV $\triangle$ G-ZEBOV-GP Ebola virus vaccine candidate in healthy adults: a phase $1 \mathrm{~b}$ randomised, multicentre, double-blind, placebo-controlled, dose-response study. Lancet Infect Dis 17: 854-866.

17. Li L, 1989. A concordance correlation coefficient to evaluate reproducibility. Biometrics 45: 255-268.

18. Lin L, Torbeck LD, 1998. Coefficient of accuracy and concordance correlation coefficient: new statistics for methods comparison. PDA J Pharm Sci Technol 52: 55-59.

19. Tan C, Iglewicz B, 1999. Measurement-methods comparisons and linear statistical relationship. Technometrics 41: 192-201. 